\title{
E-Healthcare Decision Support System based on Ontology Learning: A Conceptual Model
}

\author{
Nitesh Vyas \\ Ph.D. Scholar \\ Pacific Academy of Higher Education \& Research, \\ Udaipur, Rajasthan, India
}

\author{
P.R. Pal, PhD. \\ Professor, Department of Computer Applications \\ Ajay Kumar Garg Engineering \\ College,Ghaziabad(U.P.).
}

\begin{abstract}
E-health care decision support system is developed for the purpose of improving the health care services and control over patient's health. The system facilitates quick accessed and specialized health care services, of course, depending upon the patient's or case history by reducing the visit time to medical institutions. It provides the health care specialist a fast and up to date patient health data, thus reducing the documentation and formalities and increasing the usability of the information more effectively and reliably by the medical institutions. Healthcare institutions are designed to target the patients to deliver proper services. There is a wealth of data available within the healthcare systems but they lack effective analysis tools to discover hidden relationships and trends in data. Ontologies enable high scalability in searching, extracting, maintaining and generating information.
\end{abstract}

\section{General Terms}

Ontology, Knowledge Management, Data Driven Decision Making.

\section{Keywords}

e-healthcare, Ontology, Ontology learning, Knowledge Management, Knowledge Engineering, Protégé.

\section{INTRODUCTION}

According to WHO, health is a state of complete physical, mental and social well-being and not merely the absence of disease or infirmity. The health status is usually measured in terms of life expectancy at birth, infant mortality rate, fertility rate, crude birth rate and crude death rate.

India is a developing country it is next only to China, the second largest country in terms of population in the world. But the health status of a great majority of the people is far from satisfactory as compared to other developed countries. Health risk due to high prevalence of alcohol and tobacco consumption is also increasing. India's dream of "World Class" health care delivery system is difficult to achieve.

Hence, India faces the daunting challenge of meeting health care needs of its vast population and ensuring accessibility, efficiency, equity and quality of healthcare.

The healthcare environment is full of information related to the health but lacks in practical knowledge. It has been observed that there is an abundance of data available related to healthcare systems but it lacks effective analysis tools which helps to discover the hidden relationship and trends in data. Now a days there is a big challenge stands in front of health care organizations (hospitals and medical centers) is to provide quality service at affordable costs. Quality service implies diagnosing patients correctly and prescribed the treatments which should be effective. Poor clinical decisions can lead to disastrous consequences.
Thus e-healthcare decision support system will improve Maternal and Child health. It will reduce overall disease burden of the society. Patients have more information about their health and are more involved in the treatment process.

\section{E-HEALTHCARE}

E-healthcare is a forthcoming concept which connects the patients with doctor. It also facilitates medical Information services and provides a platform of better understanding and care.[11]

e-healthcare enable user and care giver to access user's health data anytime and anywhere. If there is e- healthcare system, user can use e-healthcare by smart home, smart care, smart office, mobile and outdoor activity. No matter where user is, user can use e-healthcare service anytime, anywhere.

"e-healtcare decision support system is a consumer-centered model of health care where the customer can use communication technologies (ICTs) including Internet technologies to manage health, arrange, deliver, and account for care, and manage the health care system". Thus e-healthcare decision support system facilitates patient to support and educate them for better health related information anytime, anywhere in the world with the flexibility of time and place

\subsection{Demographical and Related Expenditure in India :}

Current Population of India in 2012 is : 1.22 billion Age structure 0 to 25 years $50 \%$ of India's current population (As per India-current-population.html)

\begin{tabular}{|l|l|}
\hline Gross national income per capita (PPP international \$) & 2,930 \\
\hline Life expectancy at birth m/f (years) & $63 / 66$ \\
\hline Probability of dying under five (per 1 000 live births) & 66 \\
\hline $\begin{array}{l}\text { Probability of dying between 15 and 60 years m/f } \\
\text { (per 1 000 population) }\end{array}$ & $\begin{array}{l}250 / 16 \\
9\end{array}$ \\
\hline $\begin{array}{l}\text { Total expenditure on health per capita (Intl \$, } \\
\text { 2009) }\end{array}$ & 132 \\
\hline Total expenditure on health as \% of GDP (2009) & 4.2 \\
\hline
\end{tabular}

Figures are for 2009 unless indicated. Source: Global Health Observatory

\subsection{E-healthcare decision support system}

e-healthcare decision support system will be useful for both patient as well as doctor. The e-healthcare decision support system consists of:

- Centralized Server. 
- Difference server/client as different zones.

- Telemedicine

- Teleconsultation

- Electronic Health Record

- $\quad$ Smart phones.

- Mobile van.

- Academic and research institutions.

- Local health professionals and their associations.

- Consumers, patients and their associations.

- Non-governmental organizations.

- The private sector, including foundations and industries related to health and ICTs.

- E primary, secondary and tertiary care institutions, manned by medical and paramedical personnel.

- E medical colleges and paraprofessional training institutions for the training of e-heath system.

- Computer literate personal at Central as well as difference zones.

- E health management information system.

- Government authorities, health and telecommunication decision-makers at the central and in different zones.

- $\quad$ The media.

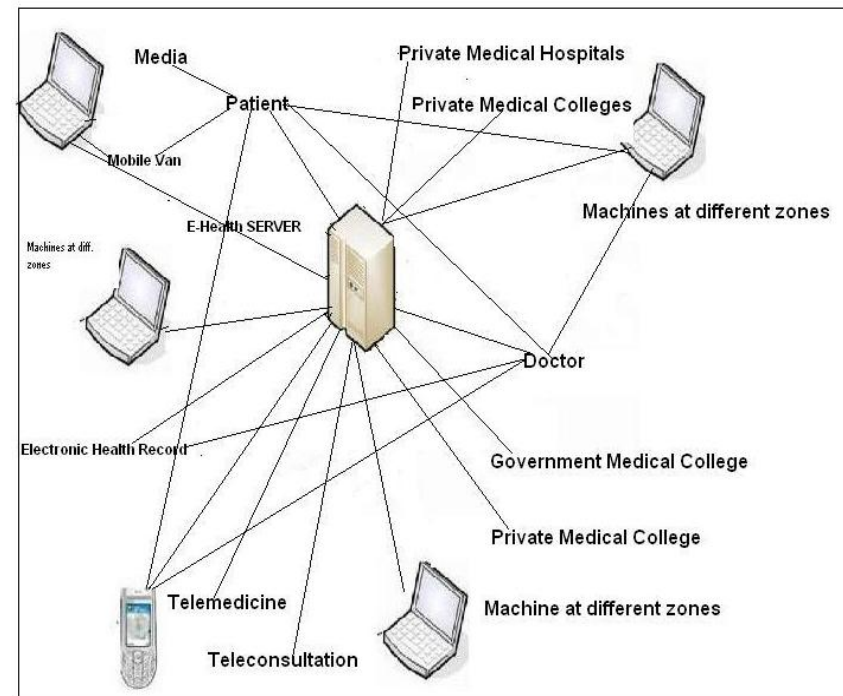

Figure 1: E-Health Decision Support System Architecture

\subsection{Electronic Health Record}

EHR will provide easier clinical data analysis. By which doctor can spent more time on patient care. With the help of EHR Physicians, hospitals, specialists and providers in the circle of care can share information quickly and easily.

\subsection{Telemedicine and Tele-consultation}

Case 1 : When Mr. $\mathrm{X}$ was an education tour (Allahabad-Banaras-Kanpur) in June 2012. Due to food poisoning, he had to take advise from his doctor through telephone. He was his family doctor so, he knows Mr. X very well. He suggest him to take following medicine : Domestral, Brakee/ Ciplox-TZ , Crosin. Again he told him another option if this medicine are not available in medical shop. After taking medicine within two hour Mr. X got well.

Following information is require for that type of telephonic talk : History : 50\%, Introduction to Problem 15\%, Introduction to Patient : 10\%, Management : $10 \%$, Investigation : $15 \%$ See figure : 2

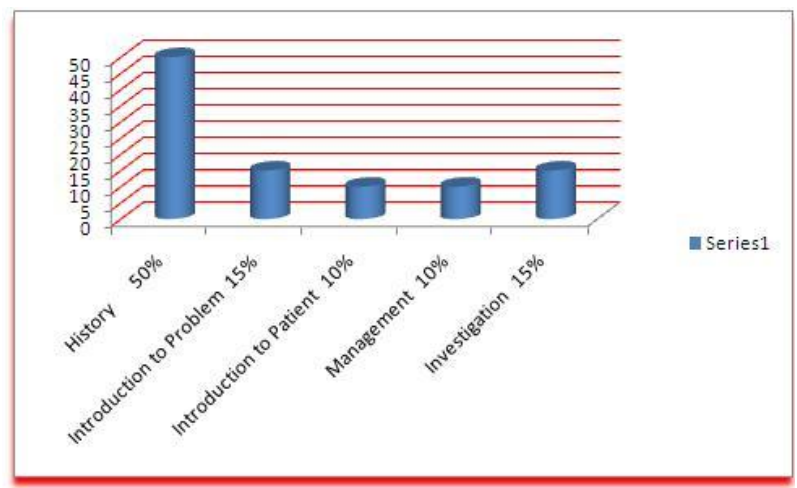

Figure 2 :Telemedicine and Tele-consultation

\section{ONTOLOGY}

The notion of ontology originates from the discipline of philosophy. It has evolved to its current meaning in the context of computer and information science where it refers to a designed arte fact which formally represents agreed semantics in a computer resource. More technically ontology is the term referring to the shared understanding of some domains of interest, which is often conceived as a set of classes (concepts), relations, functions, axioms and instances.[1] Some of most common and highly cited definition of ontology are: "An ontology is an explicit specification of conceptualization"[1] and An ontology is a formal and explicit specification of a share conceptualization"[2]. By adding two more words "formal" and "shared" the definition given by Gruber has been refined by Studer. The word formal has been added to include the sense that conceptualization is in machine understandable form. The term shared has been added to include the sense that consensual knowledge is accepted by a group. Time to time more refined definition of the term "ontology" taking other things in account has been defined. In 1995, Russel and Norving defined ontology as "Ontology is a formal description of the concepts and relations which can exist in a community of agents.’[3] In 1996 Swartout defined ontology as "Ontology is hiearchically structured set of terms to describe a domain that can be used as a skeletal foundation for a knowledge base."'[4] In 2000, Fensel defined ontology as "Ontology is common, shared and formal description of important concepts."[5] In 2001, Noy and McGuinness defined ontology as "An ontology is formal explicit representation of concepts in a domain, properties of each concept describes characteristics and attributes of the concept known as slots and constrains of these slots." [6] In 2002, 
Fonseca defined ontology as "Anontology is a theory which uses a specific vocabulary to describe entities, classes, properties and related functions with certain point of view."[7] In 2003 Starlab defined ontology as "An ontology necessarily includes a specification of the terms used, ("terminology") and agreements to determine the meaning of these terms along with the relationships between them."'[8]

Ontologies may be constructed for different purposes, for example - to enable information sharing and to support specification. When we want to enable sharing and reuse, we define an ontology as a specification used for making ontological commitments (Gruber, 1993).[1]

For example, if one is interested in health care-related knowledge, then patient, disease, symptom, diagnosis, and treatment might be among the primitive concepts upon which one might want to describe the domain. These concepts and their meanings together define an ontology for health care. Such an ontology can be used as common knowledge that facilitates communication among health workers. It can also be used during development of hospital information systems or decision-support systems.

\subsection{Ontology Buzzwords}

Few of the terms which are very common in the field of ontology are:

Concept: A concept represents a group of objects or beings sharing characteristics that enable us to recognize them as forming and belonging to this group.[9]

Term: The term is lexical representation of concept.

Ontology Engineering: Techniques for handling large ontologies.

Ontology Engineer: A person who engineers large ontologies. Ontologist: A person who builds ontologies or whose job is concerned with ontology science or engineering. [9]

Ontological Theory: A set of formulas which are intended to be always true according to a certain conceptualization.

Ontology Learning: Ontologies can be developed manually and automatically. Ontology learning focuses towards semi-automatic and automatic techniques for learning ontologies from either existing ontologies or from other data sources.

\subsection{How ontologies are relevant to healthcare}

For that answer we can use, this quote from a report titled "Semantic Interoperability Deployment and Research Roadmap" by Alan Rector, an authority in the field of biomedical ontologies: Ontologies are about the things being represented - patients, their diseases. They are about what is always true, whether or not it is known to the clinician. For example, all patients have a body temperature (possibly ambient if they are dead); however, the body temperature may not be known or recorded. It makes no sense to talk about a patient with a "missing" body temperature.

Medical ontologies are developed to solve problems such as the demand for reusing and sharing patient data or the transmission of these data. The unambiguous communication of complex and detailed medical concepts is a crucial feature in current medical information systems. In these systems, several agents must interact in order to share their results and, thus, they must use a medical terminology with a clear and non-confusing meaning [10][12].

The development of these ontologies is a complex task: on the one hand, they are general enough to be required for achieving consensus between a wide community of users and, on the other hand, they are concrete enough to present an enormous diversity with hundreds of possible concepts to model.

Medical ontology engineering is typically performed manually, requiring the intervention of medical specialists (which provide the medical knowledge) and knowledge engineers (which are able to formalize that knowledge). The required consensus is typically hampered by the difficulty of translating the shared world model of a medical community to the formal and explicit knowledge representation of an ontology. This produces long and tedious development stages that delay the applicability of the resulting ontologies.

Due to all these reasons, nowadays, there is a need of methods that can perform, or at least ease, the construction of medical ontologies. In this sense, Ontology learning is defined as the set of methods and techniques used for building from scratch, enriching, or adapting an existing ontology in a semiautomatic fashion using distributed and heterogeneous knowledge and information sources [10][12]. These methods allow a reduction in the time and effort needed in the ontology development process.

Ontology learning is a subtask of information extraction. The goal of ontology learning is to semi automatically extract relevant concepts and relations from a given corpus or other kind of data sets to form an ontology.

Ontology learning is also one of the most prominent area of research where semiautomatic and automatic techniques of learning ontology from various kind of data sources are explored. A general overview of ontology learning from text has been shown in figure 3 .

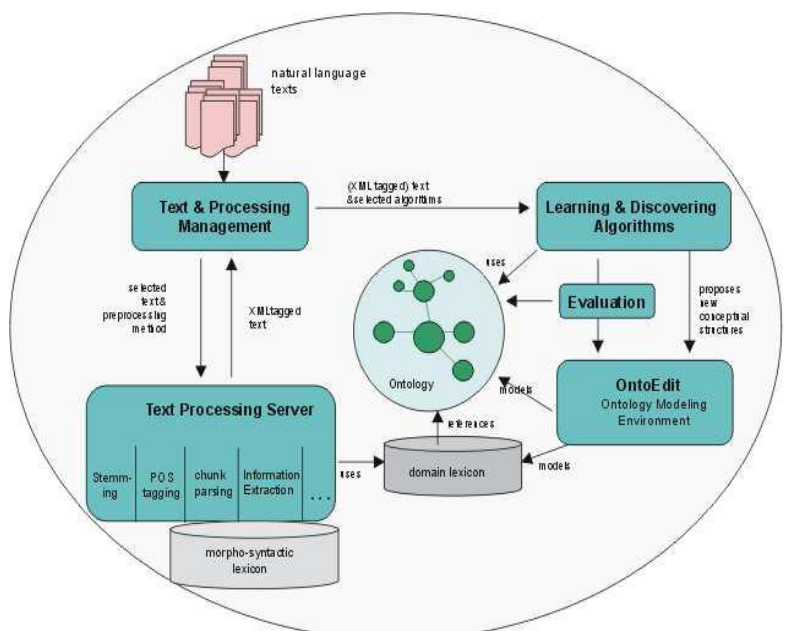

Figure 3: General overview of Ontology learning System Text-to-Onto

Learning ontologies from relational schemata is opening new era of developing knowledge based intelligent system. ontology based decision making projects. The Shah laboratory studies ontology based approaches to annotate, index, integrate and analyze diverse information types available in 
biomedicine for the purpose of enabling datadriven decision making in medicine and health care. [14]

\section{KNOWLEDGE MANAGEMENT}

Efficient knowledge management is very essential for an organization to have greater productivity and competitive strength. In this fast contemporary era, competitiveness among organizations depends largely on how they exploit and maintain their knowledge. This is the age of Information Technology. Today, information is available everywhere. Earlier news papers were very good source of information. The advancement in science and technology, has resulted in enhancing a wider range of options. Radio \& Television are the major audio and visual sources of getting information. Now even, the news papers are available on the Internet. Most of the information in Intranet or Internet is weakly structured. Finding relevant information and maintaining it is difficult. If the information on the Internet is not utilized effectively it is useless. In most of the knowledge management systems, the search is based on keywords and the chance of irrelevant information due to keyword ambiguity is high. Software agents used in extracting information do not have common sense to assist effectively in the search. Also they are unable to integrate information from different sources. Ontologies enable high scalability in searching, extracting, maintaining and generating information. Figure 4 shows the knowledge management in healthcare system :

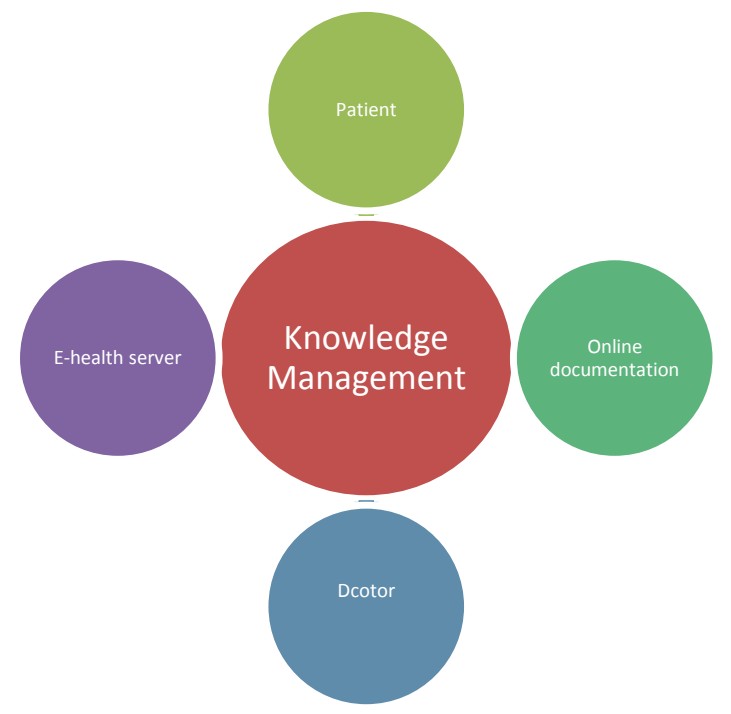

Figure 4: Knowledge Management in Healthcare System

\section{KNOWLEDGE ENGINEERING}

Relational databases are well suitable for modeling data required by information system. Information systems are used to manage the systems especially for automatic electronic data processing, taking decisions based on the data to achieve a goal. Processed data gives Information. Information changes in to the Knowledge. Knowledge again changes to Intelligence. With the current shape of data of information system it is quite impossible to convert information to knowledge and then to intelligence for the real time need of system users.

Looking towards this problem a new area entitled by Knowledge Engineering has come into picture. Knowledge engineering is an engineering discipline that involves integrating knowledge in computer systems in order to solve complex problems normally requiring a high level of expertise.

Ontology extraction would be a key component of such information architecture. Ontology language has a clear and unambiguous semantics which enables the use of automatic reasoning support to designer. For a special purpose application a schema is designed on the basis of requirement of data. Ontologies extracted from the Relational Schemata and updations in it on the basis of dynamic population of data prompt the process of learning for the ontologies to help automatic decision making.

Tools available for building the suitable ontology structure for e-healthcare solution :

Karlsruhe Ontology Framework (KAON) : It is an open source ontology management and learning infrastructure targeted for business applications. It includes a comprehensive tool suite allowing easy ontology creation supported by machine learning algorithms.

FrameLogic: They provide a visual metaphor for representing the conceptual structure.

Description logic (DLs) is one most popular framework at the moment. DLs are subsets of first order logic which aim at being as expressive as possible while still being decidable.

Prot'eg'e-2000 is a platform-independent environment for creating and editing ontologies and knowledge bases. Like $\mathrm{KAON}$, it has an extensible plug-in structure.

Sesame is architecture for efficient storage and expressive querying of large quantities of $\mathrm{RDF}(\mathrm{S})$ data.

\section{BENEFITS}

- Patients will have more information about their health and more involved in the treatment process.

- Process allows for transparency as unfair practices are easily detected.

- e-Health supports the patients to obtain better care, prevent medical mistakes and have saved their own lives at times.

- $\quad$ EHCSM will help us drive down wait times, reduce duplication and unnecessary tests, and put a stop to avoidable prescription drug errors.

- Improve Maternal and Child health.

- It will reduce overall disease burden of the society.

- Strengthening Secondary and Tertiary health care.

- Ultimately, it will save lives, improve care and save significant amounts of money.

\section{SUGGESTION AND CHALLENGES}

- Centralized system like Indian railway and it should be divide in different zones like : East_medical 
zone, West_medical zone, North_medical zone, South_medical zone etc.

- According to the latest report released by Telecom Regulatory Authority in India (TRAI), the overall Tele-density in Indian reaches 67.67 percent. So, mobile medical system will change the life cycle of Indian people.

- Development of National Plan for e-health.

- Appropriate e-health system for local situation with global impact

- BSNL is everywhere so, it will be helpful in e-healthcare system.

- $\quad$ Fund will be the major problem.

- Most of villagers doesn't know about the internet. So, Computer illiteracy is also one of the another problem.

\section{CONCLUSIONS}

By using e-health care systems, patients will receive better, faster and more coordinated health care from their providers and providers will be able to share information more effectively. The system will help in handling track record of patients. Ontology design is a creative process and the automated role of doctor by using efficient ontologies in the system may entirely change the clinical practice. This e-health solution model will be helpful for both doctors as well as patient in providing high quality service in low cost.

\section{FUTURE WORK}

As further work we plan to consider the issue of complex reasoning in E-health care systems require the task to be achieved. OWL in combination with SWRL can be used for handling in reasoning in complex medical systems. Another bigger issue that need to be taken care is automatic construction of ontologies from the clinical database so that learning process of e-health care system can be continued with the passage of time.

\section{REFERENCES}

[1] T.R. Gruber. A translation approach to portable ontologies. Knowledge Acquisition, 5(2):199-220, 1993

[2] Runi Studer, V. Richard Benjamins, Dieter Fensel: Knowledge Engineering: Principles and Methods. Data Knowl. Eng. 25(1-2): 161-197 (1998)
[3] Russell S., Norvig P. Artificial Intelligence: A Modern Approach. Prentice Hall, Englewood Cliffs, NJ. (1995)

[4] Swartout B., Patil R., Knight K., Russ T. Toward distributed use of large-scale ontologies. In Proceedings of the Tenth Knowledge Acquisition for KnowledgeBased Systems Workshop. (KAW'96 November 9-14, Banff, Alberta, Canada). (1996)

[5] Fensel D., 2000. The semantic web and itslanguages. IEEE Computer Society 15, 6 (November/December), 67-73.

[6] Noy N.F., McGuinnes D. L. 2001. “'Ontology Development 101: A Guide to Creating Your First Ontology'. Stanford Knowledge Systems Laboratory Technical Report KSL-01-05 and Stanford Medical Informatics Technical Report SMI-2001-0880, March.

[7] Fonseca, F. Egenhofer M., Agouris, P., Camara G. 2002. Using Ontologies for Integrated Geographic Information Systems. Transactions in GIS, $-(6): 3$ in print.

[8] Starlab 2003. Systems Technology and Applications Research Laboratory home page. Faculty of Sciences, Department of Computer Science, Vrije UniversiteitcBrussel. Available at http://www.starlab.vub.ac.be/default.htm

[9] Fabien Gandon "Distributed Artificial Intelligence And Knowledge Management: Ontologies And Multi-Agent Systems For A Corporate Semantic Web", scientific philosopher doctorate thesis in informatics INRIA and University of Nice- Sophia Antipolis- Doctoral School of Sciences and Technologies of Information and Communication (S.T.I.C) 7/11/2002, 486 pages.

[10] Gómez-Pérez, A., Fernández-López, M., Corcho, O. Ontological Engineering, Springer Verlag, 2nd printing. (2004)

[11] Mr. Manish Kumar Khare, Ontology based machine learning using data mining techniques for intelligent decision making in healthcare sector, Symboisis International Deemed University, Pune

[12] Learning medical ontologies from the Web David Sánchez, Antonio Moreno Intelligent Technologies for Advanced Knowledge Acquisition (ITAKA) Research Group Department of Computer Science and Mathematics Universitat Rovirai Virgili (URV)

[13] http://bmir.stanford.edu/labs/view.php/shah_lab 